\title{
Diffusion Tensor Imaging Reliably Detects Experimental Traumatic Axonal Injury and Indicates Approximate Time of Injury
}

\author{
Christine L. Mac Donald, ${ }^{1}$ Krikor Dikranian, ${ }^{3}$ Philip Bayly, ${ }^{1,2}$ David Holtzman, ${ }^{3,45,6}$ and David Brody ${ }^{4,6}$ \\ Departments of ${ }^{1}$ Biomedical Engineering and ${ }^{2}$ Mechanical Engineering, Washington University, St. Louis, Missouri 63130, Departments of ${ }^{3}$ Anatomy and \\ Neurobiology, ${ }^{4}$ Neurology, and ${ }^{5}$ Molecular Biology and Pharmacology, and ${ }^{6}$ Hope Center for Neurological Disorders, Washington University, St. Louis, \\ Missouri 63110
}

Traumatic axonal injury (TAI) may contribute greatly to neurological impairments after traumatic brain injury, but it is difficult to assess with conventional imaging. We quantitatively compared diffusion tensor imaging (DTI) signal abnormalities with histological and electron microscopic characteristics of pericontusional TAI in a mouse model. Two DTI parameters, relative anisotropy and axial diffusivity, were significantly reduced $6 \mathrm{~h}$ to $4 \mathrm{~d}$ after trauma, corresponding to relatively isolated axonal injury. One to 4 weeks after trauma, relative anisotropy remained decreased, whereas axial diffusivity "pseudo-normalized" and radial diffusivity increased. These changes corresponded to demyelination, edema, and persistent axonal injury. At every time point, DTI was more sensitive to injury than conventional magnetic resonance imaging, and relative anisotropy distinguished injured from control mice with no overlap between groups. Remarkably, DTI changes strongly predicted the approximate time since trauma. These results provide an important validation of DTI for pericontusional TAI and suggest novel clinical and forensic applications.

Key words: magnetic resonance imaging; diffusion tensor imaging; traumatic brain injury; axonal injury; white matter injury; forensic imaging

\section{Introduction}

Traumatic axonal injury may be a major contributor to cognitive dysfunction in patients after traumatic brain injury (TBI) (Strich, 1956, 1961; Nevin, 1967; Oppenheimer, 1968; Adams et al., 1982; Gennarelli et al., 1982; Pilz, 1983; Povlishock et al., 1992; Smith et al., 2003; King et al., 2005). Current clinical imaging modalities, including computed tomography and conventional magnetic resonance imaging (MRI), have been optimized for evaluating hemorrhage, edema, and ischemia but are inadequate for direct assessment of injured axons (Silver et al., 2005). Diffusion tensor imaging (DTI) has been proposed as a noninvasive method to directly detect and characterize axonal injury (Werring et al., 1998; Rugg-Gunn et al., 2001; Arfanakis et al., 2002; Ptak et al., 2003; Song et al., 2003; Huisman et al., 2004; Naganawa et al., 2004; Inglese et al., 2005; Nakayama et al., 2006; Voss et al., 2006; Wilde et al., 2006). DTI measures water diffusion in many independent directions. Because of its highly organized structure, water diffusion in normal white matter is highly anisotropic (direc-

Received May 16, 2007; accepted Sept. 11, 2007.

This work was supported by National Institutes of Health Grants NS049237 (D.B.), P01 NS032636 (P.B.), R21 NS45237 (P.B.), and P30 NS057105 (D.H.) and a Burroughs Wellcome Career Award in the Biomedical Sciences (D.B.). We acknowledge Dr. John Olney for the use of his electron microscope and for insightful discussions regarding electron microscopy. We also acknowledge Drs. Mary Case, Jeff Neil, Victor Song, and Kurt Thoroughman for helpful scientific discussions.

Correspondence should be addressed to Dr. David Brody, Department of Neurology, Washington University, 660 South Euclid Avenue, Box 8111, St. Louis, M0 63110. E-mail: brodyd@neuro.wustl.edu.

DOI:10.1523/JNEUROSCI.3647-07.2007

Copyright $\odot 2007$ Society for Neuroscience $\quad$ 0270-6474/07/2711869-08\$15.00/0 tionally asymmetric); water diffuses more readily parallel to the predominant local orientation of axons than it does perpendicular to this predominant direction. When axons are injured, water diffusion may change such that anisotropy decreases dramatically, whereas overall diffusivity changes very little. Encouragingly, DTI studies in TBI patients have shown white matter signal abnormalities not observed with conventional MRI (Rugg-Gunn et al., 2001; Arfanakis et al., 2002; Huisman et al., 2004; Inglese et al., 2005). However, this technique has not been fully validated, in that few direct comparisons with histologically verified axonal injury have been performed.

Our initial studies in a mouse model indicated that DTI is, in fact, sensitive to histologically verified axonal injury in regions where conventional imaging methods were not (Mac Donald et al., 2007). These studies were performed at only a single time point, $24 \mathrm{~h}$ after injury. It is unknown whether there are changes in the sensitivity of DTI over time, because only very limited clinical studies (Arfanakis et al., 2002; Naganawa et al., 2004; Voss et al., 2006) and none of the previous experimental animal studies (Hanstock et al., 1994; Kochanek et al., 1995; Alsop et al., 1996; Assaf et al., 1997; Albensi et al., 2000; Duhaime et al., 2003; Van Putten et al., 2005), to our knowledge, have performed DTI at multiple time points. For optimal clinical utility, a method should be robust across a variety of time points, given that it is rarely possible to scan patients at a fixed time point after injury.

Here we show that DTI is highly sensitive to experimental pericontusional white matter injury throughout the full range of 
time points studied and, in fact, is consistently more sensitive than conventional MRI. Surprisingly, we also found that DTI signal changes could distinguish the approximate time since injury. If similar in human TBI, this could allow novel forensic applications.

\section{Materials and Methods}

Experimental design. For these experiments, a total of 30 male and female 8- to 10-week-old B6SJL F1 mice (The Jackson Laboratory, Bar Harbor, ME) were used. One mouse died during imaging, and 29 mice were included in the analyses. Five separate parallel groups of four to five mice per group were injured and imaged at $4-6$ h, 24 h, 4 d, 1 week, or 1 month after injury. Mice in each group were killed for histology immediately after the scan. A control group of six mice was imaged and killed for histology but not injured. All procedures involving animals were approved by the Washington University Animal Studies Committee and were consistent with National Institutes of Health guidelines for the care and use of animals.

Experimental TBI: controlled cortical impact. The experimental TBI methods used for this study have been described previously (Brody et al., 2007; Mac Donald et al., 2007). Briefly, mice were anesthetized with isoflurane and placed in a stereotaxic frame (MyNeuroLab, St. Louis, $\mathrm{MO})$. Using sterile technique, a midline incision was made, and the scalp was reflected to expose the skull. A $5 \mathrm{~mm}$ left lateral craniotomy was performed using a motorized drill mounted to the stereotactic arm. A controlled cortical impact injury was produced with an electromagnetic impact device mounted on a stereotaxic arm (Brody et al., 2007). The impact was centered at $2.7 \mathrm{~mm}$ lateral from midline and $3 \mathrm{~mm}$ anterior from lambda. The impact depth was set at $2.5 \mathrm{~mm}$ using the stereotaxic device. The impactor was then driven at a velocity of $5 \mathrm{~m} / \mathrm{s}$ with a dwell time of $100 \mathrm{~ms}$. This produces a moderately severe contusion in the left sensorimotor cortex and underlying hippocampus with pronounced behavioral deficits but virtually no mortality (Brody et al., 2007). After injury, a plastic skull cap was secured over the impact site, the skin incision was sutured closed, and the animals were allowed to fully recover.

Image acquisition. DTI images were acquired as described previously using a 4.7T scanner [Oxford Instruments (Oxfordshire, UK) 200/330] under isoflurane anesthesia. Identical anatomical markers were used for all imaging sessions to obtain anatomically matched coronal images at each time point. A multislice, spin-echo imaging sequence, modified to include the Stejskal-Tanner diffusion sensitizing gradient pair, was used to acquire the diffusion-weighted images (Song et al., 2004). These images were acquired with a repetition period (TR) of $3 \mathrm{~s}$, a spin-echo time (TE) of $43 \mathrm{~ms}$, a time between application of gradient pulses $(\Delta)$ of $25 \mathrm{~ms}$, a diffusion gradient duration $(\delta)$ of $10 \mathrm{~ms}$, a slice thickness of $0.5 \mathrm{~mm}$, a field of view of $2.0 \mathrm{~cm}$, a data matrix of $128 \times 128$, and zero filled to $256 \times$ 256 . This resulted in a voxel size of $78 \mu \mathrm{m} \times 78 \mu \mathrm{m} \times 0.5 \mathrm{~mm}$ after zero fill. This voxel size was chosen because it allowed clear visualization of the boundaries between gray and white matter on coronal slices (see Fig. 4). Diffusion sensitizing gradients were then applied along six directions: $[G x, G y, G z]=[1,1,0],[1,0,1],[0,1,1],[-1,1,0],[0,-1,1]$, and $[1$, $0,-1]$. The two diffusion sensitizing factors or $b$ values used for acquisition of the diffusion-weighted series were 0 and $764 \mathrm{~s} / \mathrm{mm}^{2}$. The total imaging time was $\sim 3 \mathrm{~h}$ per scan.
TBI: Acute

$\mathbf{B}$

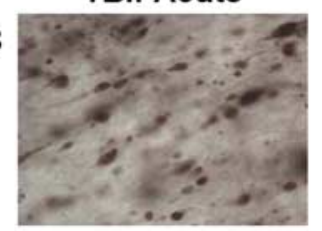

E

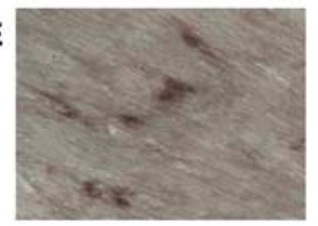

H

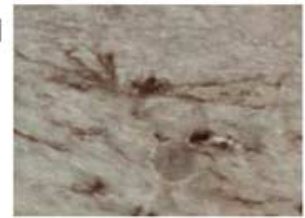

K

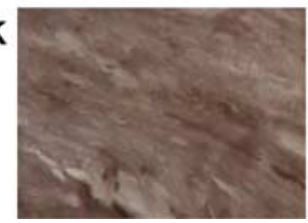

TBI: Subacute

C

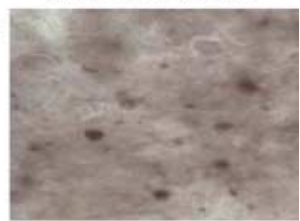

F

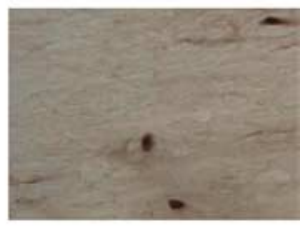

I

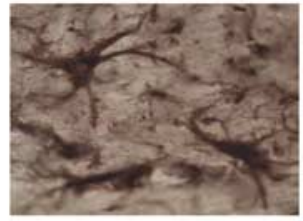

L

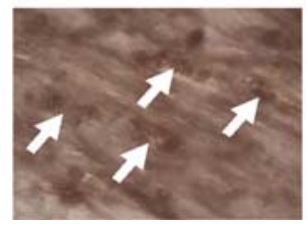

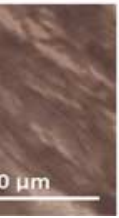

gure 1. Pericontusional white matter histopathology after experimental controlled cortical impact TBl in mice. APP $(\boldsymbol{A}-\boldsymbol{C})$ $\boldsymbol{G}$, Uninjured white matter shows no APP, NF-L, or GFAP staining in the corpus callosum and external capsule. $\boldsymbol{B}, \boldsymbol{E}$, Acutely after

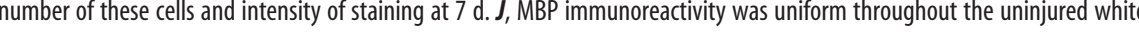
matter. $\boldsymbol{K}$, Acutely after injury, MBP immunoreactivity appeared similar to control. $\boldsymbol{L}$, Subacutely, MBP immunoreactivity was stil

Definition of a region of interest containing axonal injury in corpus callosum and external capsule. The anatomical extent of axonal injury in the corpus callosum and external capsule was described based on the amyloid precursor protein (APP)-stained histological slides, as detailed previously (Mac Donald et al., 2007). In the most rostral regions displaying a complete corpus callosum (bregma, +1.10 to $-0.34 \mathrm{~mm}$ ) (Franklin and Paxinos, 1997), the boundaries were the midline and the lateral edge of the cingulum. Moving more caudally (bregma, -0.34 to $-1.06 \mathrm{~mm}$ ), the region of interest (ROI) included the corpus callosum and external capsule from midline to a boundary defined by a horizontal line extending laterally from the bottom of the fimbria until it intersected with the external capsule (see Fig. 4). The ROI in the most caudal slices (bregma, -1.06 to $-3.08 \mathrm{~mm}$ ) included the corpus callosum and external capsule from midline to a boundary defined by a similar horizontal line extending laterally from the lateral-inferior edge of the hippocampus. All chosen anatomical landmarks were clearly visible on MR images.

Analysis of DTI images using anatomically defined ROIs. Analysis was performed with NIH ImageJ software. The ROI was traced on both the ipsilateral and contralateral sides through nine coronal slices for each control and post-TBI T2-weighted image set. The ImageJ software binding feature allowed for the simultaneous replication of the traced ROI to the other image sets. These included relative anisotropy (RA), axial diffusivity (AD), radial diffusivity (RD), and mean diffusivity or apparent diffusion coefficient (ADC) as defined previously (Mac Donald et al., 2007). At the epicenter of the injury, there was disruption of cortex, white matter tracts, and hippocampus; signal abnormality was apparent on all of the imaging modalities. The epicenter was omitted from the ROI, and only the remaining pericontusional white matter was traced. The ImageJ 

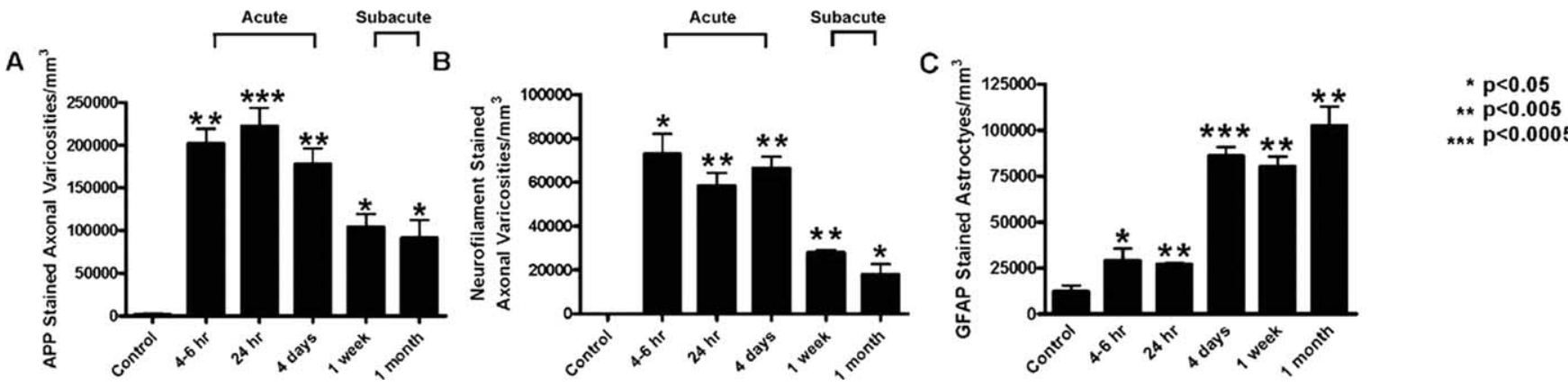

Figure 2. Stereological quantification of axonal injury and gliosis after TBI. $\boldsymbol{A}$, Estimated numbers of APP-stained axonal varicosities per cubic millimeter as a function of time after injury. There was a statistically significant increase in APP-stained axonal varicosities indicative of axonal injury at all time points after trauma, with the greatest increases at the acute time points (4-6 $\mathrm{h}, 24 \mathrm{~h}$, and 4 d). $\boldsymbol{B}$, Estimated numbers of neurofilament light chain (NR4 antibody)-stained axonal varicosities per cubic millimeter as a function of time after injury. Again, there was a statistically significant increase in neurofilament light chain-stained immunoreactive axonal varicosities at all time points after injury, and these changes were more marked at the acute time points than at the subacute time points. C, Estimated numbers of GFAP-stained immunoreactive astrocytes per cubic millimeter as a function time after injury. Compared with control, there was a significant elevation at all time points after injury; the numbers of immunoreactive astrocytes rose markedly at $4 \mathrm{~d}$ after injury and remained elevated at $7 \mathrm{~d}$ and 1 month. Error bars represent SDs. $n=4-6$ mice per group. Statistical significance was determined with a Student's $t$ test for independent samples compared with control.

program then returned the average signal intensity for each traced subregion of interest on each slice. The average signal intensity for the complete ROI was obtained by calculating the average across all slices weighted by the fraction of total voxels in each slice.

Stereological quantification of histological features of injury. After imaging, mice were killed, and brains were removed, fixed, equilibrated in sucrose, sliced, and stained as described previously (Mac Donald et al., 2007). For these experiments, the following antibodies were used for immunohistochemistry: polyclonal rabbit anti- $\beta$-APP (Invitrogen, Carlsbad, CA) in a 1:500 dilution; monoclonal mouse antineurofilament 68 clone NR4 (Sigma, St. Louis, MO) in a 1:500 dilution; polyclonal chicken anti-glial fibrillary acidic protein (GFAP) (Chemicon, Temecula, CA) in a 1:1000 dilution; or monoclonal rat anti-myelin basic protein (Chemicon) in a 1:500 dilution. All were incubated overnight at $4^{\circ} \mathrm{C}$. Secondary antibodies (Vector Laboratories, Burlingame, CA) used were biotinylated goat anti-rabbit antibody $(\beta$-APP), goat anti-mouse IgG antibody (neurofilament), donkey anti-chicken antibody (GFAP), or goat anti-rat antibody (myelin basic protein). All secondary antibodies were incubated in a 1:1000 dilution for $1 \mathrm{~h}$ at room temperature, and the slides were developed with 3,3' -diaminobenzidine tablets (Sigma).

Stereological methods were used to quantify the numbers of APP- or neurofilament-stained axonal varicosities and GFAP-stained immunoreactive astrocytes per cubic millimeter in the ROIs (Sterio, 1984). This analysis was performed using StereoInvestigator 6.0 (MicroBrightField, Williston, VT) and a Nikon (Tokyo, Japan) Eclipse E800 microscope. The Gunderson coefficient of error $(m=1)$ was maintained below 0.05 for each set. Counts were performed by a blinded observer. The optical fractionator technique was used to count a systematic random sample of positively stained axonal varicosities or astrocytes over the entire rostral to caudal extent of the ROIs. For each coronal slice from each animal, the ROI was outlined at low power $(4 \times)$ followed by systematic counts of objects of interests at high power $(60 \times$, oil immersion) over sites within the ROI randomly chosen by the StereoInvestigator software. A $40 \times 40$ $\mu \mathrm{m}$ counting frame was used, and a $15-\mu \mathrm{m}$-deep region was sampled. Injured axons were defined by the presence of APP- or neurofilamentstained varicosities that were $\geq 6 \mu \mathrm{m}$ in diameter and were in focus within the 15 - $\mu \mathrm{m}$-deep counting region, beyond the $2.5 \mu \mathrm{m}$ guard zone. Red blood cells and other nonaxonal-stained structures were not counted. GFAP-immunoreactive astrocytes were counted if their cell bodies were within the counting frame and in focus in the counting region.

The volumes of the complete ROIs were calculated using the Cavalieri principle; the area of the ROI in each sampled slice was multiplied by the distance between sampled slices, and the resultant volumes were summed. The total volume of the sampled regions was calculated by multiplying the area of each counting frame $\left(1600 \mu \mathrm{m}^{2}\right)$ by the thickness of the sampled region $(15 \mu \mathrm{m})$ and then multiplying by the number of counting frames sampled, which varied from animal to animal. To esti- mate the total number of axonal varicosities or immunoreactive astrocytes within the complete ROI, the number of each entity counted was multiplied by the volume of the ROI and divided by the volume sampled. To estimate the number of axonal varicosities per cubic millimeter or astrocytes per cubic millimeter, the estimated total counts of each object were divided by the volumes of the sampled regions.

Electron microscopy. For electron microscopy studies, animals were anesthetized and perfused transcardially with a fixative composed of $1.5 \%$ glutaraldehyde and $1 \%$ paraformaldehyde in cacodylate buffer, $\mathrm{pH}$ 7.4. The brains were sliced coronally into $1 \mathrm{~mm}$ slabs. These slabs were osmicated overnight ( $1 \%$ osmium tetroxide) at room temperature, dehydrated in graded ethanols, cleared in toluene, and embedded flat in Araldite resin. Thin sections, $1 \mu \mathrm{m}$ thick, were cut at selected rostrocaudal levels of the brain, using glass knives (1/2 inch wide) and an MT-2B Sorval ultramicrotome. These sections were heat dried on glass slides and stained with methylene blue azure II for evaluation of areas of special interest by light microscopy. For electron microscopy, the tissue around the areas of special interest was trimmed, ultrathin $(75-85 \mathrm{~nm})$ sections were cut using a Leica (Nussloch, Germany) Ultracut E microtome, and sections were mounted on formvar-coated grids. Grids were then stained with uranyl acetate and lead citrate. Ultrastructural observation was performed using a Jeol (Tokyo, Japan) 100C transmission electron microscope.

Statistical methods. All data were analyzed using Statistica 6.0 (StatSoft, Tulsa, OK). Quantitative results from corresponding ROIs were compared between the control and posttrauma histological and imaging data sets. Unpaired $t$ tests were used because there was no evidence for deviation from the normal distribution (Shapiro-Wilks $W$ test) for any of the parameters. Prespecified hypotheses were that the numbers of histological markers of injury (APP, neurofilament, GFAP) would increase after TBI and the DTI parameters RA and AD would decrease after TBI, so one-sided $t$ tests were used for these comparisons. For the MRI parameters $\mathrm{RD}, \mathrm{ADC}$, and $\mathrm{T} 2$, there were no prespecified hypotheses about the direction of change, so two-sided $t$ tests were used. For comparisons between acute $(0-4 \mathrm{~d})$ and subacute ( 1 week to 1 month) groups, the data from the groups imaged at $4-6 \mathrm{~h}, 24 \mathrm{~h}$, and $4 \mathrm{~d}$ were pooled, and the data from groups imaged at 1 week and 1 month were pooled. These two pooled sets were then compared with each other using two-sided $t$ tests because there were no prespecified hypotheses about the direction of changes. The threshold for statistical significance was set to $p<0.05$ without correction for multiple comparisons.

\section{Results}

Histological and EM analysis of pericontusional traumatic axonal injury in a mouse model

Moderately severe controlled cortical impact TBI in adult mice produced a central contusion in cortex and hippocampus, and 
extensive pericontusional injury in the corpus callosum and external capsule. We characterized the time course of histological changes in this pericontusional white matter injury to generate hypotheses about the expected DTI imaging features at each time. Importantly, axonal injury was detected in the corpus callosum and external capsule at all time points after injury (Fig. 1). Greater numbers of APP- or neurofilament-stained, injured axonal varicosities were present at the acute time points $(0-4 \mathrm{~d})$ than at the subacute (7-30 d) time points (Fig. $1 A-F)$. Immunoreactive astrocytes were also observed in the corpus callosum and external capsule at all time points, but with greater numbers at the subacute time points (Fig. 1G-I). There was no staining for either marker of axonal injury, and there were few immunoreactive astrocytes in uninjured mice. Myelin basic protein immunohistochemistry revealed relatively uniform staining in the injured white matter at acute time points that was similar to control (Fig. $1 \mathrm{~J}, \mathrm{~K})$. At the subacute time points, the myelin staining appeared "clumpy," and spheroidal structures were noted (Fig. $1 \mathrm{~L}$, white arrows). Frank myelin pallor was not consistently observed at any time point. No staining was observed when the primary antibodies were omitted (data not shown).

These histological results were quantified using stereological techniques (Fig. 2). ROIs defining the pericontusional white matter injury (see Materials and Methods) were outlined, and random sampling was performed to allow unbiased estimation of the numbers of each immunohistochemically stained structure. A significant increase in the numbers of APP- or neurofilamentstained axonal varicosities was observed at all time points after injury (Fig. $2 A, B$ ). More APP- or neurofilament-stained axonal varicosities were apparent at the acute time points compared with the subacute time points. The two markers of axonal injury, APP and neurofilament, revealed similar temporal trends (Fig. 2A,B). Numbers of GFAP-immunoreactive astrocytes (Fig. 2C) were also significantly elevated. However, the time course was different from that of axonal injury; relatively modest numbers of GFAP-stained astrocytes were present at $4-6$ and $24 \mathrm{~h}$, then their numbers increased dramatically starting $4 \mathrm{~d}$ after injury.

Electron micrographs confirmed the presence of axonal injury and gave further insight into the differences in pathology between the acute and subacute stages (Fig. 3). At acute time points, APPstained semithin sections counterstained with methylene blue/ azur allowed localization of regions containing injured axonal varicosities within the corpus callosum (Fig. 3A). Electron micrographs of these regions revealed organelle compaction within myelinated axonal varicosities (Fig. $3 B$ ), small regions of focal myelin loss (Fig. $3 B, C$ ), neurofilament compaction (Fig. $3 B, C$ ), and cytoskeletal abnormalities including disruption of microtubules (Fig. $3 D, E$ ). There was distortion of mitochondria (Fig. $3 E$ ), and in some regions, severe intracellular and extracellular edema was observed (Fig. $3 F$ ).

At the subacute time points, the white matter of the corpus callosum and external capsule was invaded by phagocytic cells (Fig. 3G). Electron micrographs revealed that the cells were macrophages, some of which had phagocytosed damaged axons, many still myelinated (Fig. $3 \mathrm{H}$ ). A higher-magnification electron micrograph (Fig. 3I) taken of a phagocytosed, myelinated axon shows the swollen rings of myelin surrounding the axoplasm and intra-axonal mitochondrial damage. This finding sheds light onto the "clumpy," spheroidal structures noted at the subacute time points with myelin staining (Fig. $1 \mathrm{~L}$ ); these are likely to be macrophages that have ingested degenerating myelinated axons. Advanced stages of axoplasmic degeneration were seen in deteriorating axons (Fig. 3J) along with myelin thinning and marked

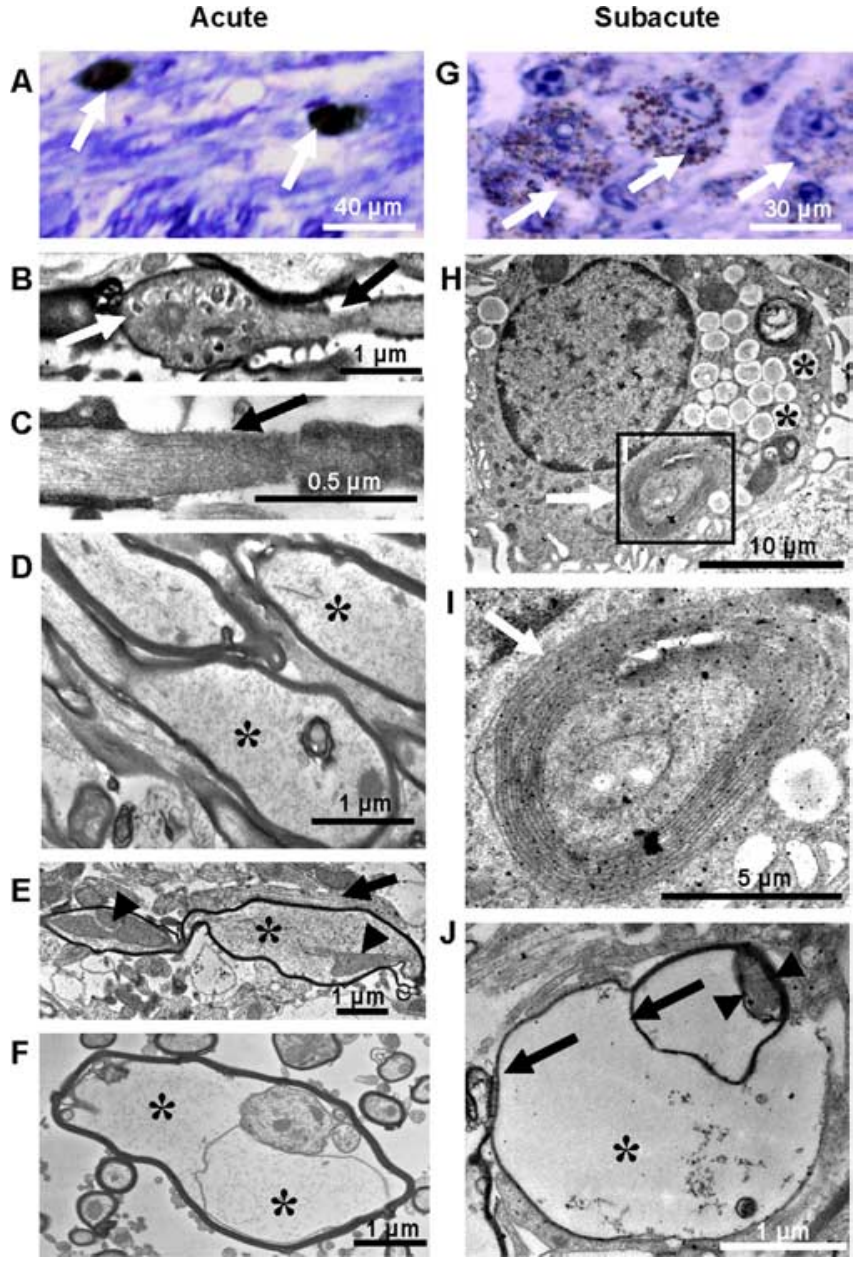

Figure 3. Ultrastructural changes at acute $(\boldsymbol{A}-\boldsymbol{F})$ and subacute $(\boldsymbol{G}-\boldsymbol{J})$ time points after TBI. $\boldsymbol{A}, \mathrm{APP}$ immunohistochemistry in a semithin section counterstained with methylene blue/azur. Regions containing APP-stained axonal varicosities (white arrows) were used to guide EM analysis. $\boldsymbol{B}$, Nodal segment of an injured axon in the corpus callosum demonstrating organelle compaction (white arrow) within a varicosity. Note the generally preserved myelin sheathes. An area of focal myelin loss with damaged axonal cytoskeletal structures (black arrow) adjacent to the varicosity is shown. $\boldsymbol{C}$, Axonal segment with neurofilament compaction and focal myelin loss (black arrow). D, Longitudinal section through a swollen segment of a myelinated axon in the external capsule, rostral to the epicenter of injury, with breakdown of cytoskeletal filaments and lack of microtubules (asterisks). $\boldsymbol{E}$, Two connected varicosities with distorted mitochondria (arrowheads) and disrupted cytoskeletal structures (asterisk) in a myelinated axon. Note an adjacent segment of an intact unmyelinated axon (black arrow). $\boldsymbol{F}$, Cross section of a myelinated axon from a severely injured region deformed by edema (asterisks). G, Semithin section stained with methylene blue/azur from a region of the subacutely injured corpus callosum invaded by phagocytic cells (white arrows). $\boldsymbol{H}$, Macrophage in the corpus callosum showing intracytoplasmic vacuoles (asterisks) and a degenerating phagocytosed segment of a markedly swollen, myelinated axon (white arrow). I, High-magnification image of the degenerating axon phagocytosed by the macrophage in $\boldsymbol{H}$ (white arrow). $\boldsymbol{J}$, Advanced stage of axoplasmic degeneration (arrowheads) in a myelinated axon in the external capsule showing myelin thinning (black arrows) and marked intracellular edema (asterisk).

intracellular edema. Additional electron micrographs are shown in supplemental Figs. 1 and 2 (available at www.jneurosci.org as supplemental material). Intra-axonal disruption with little loss of myelin sheaths was consistently observed $24 \mathrm{~h}$ after injury (supplemental Fig. 1, available at www.jneurosci.org as supplemental material). At $7 \mathrm{~d}$ after trauma, myelin thinning and marked edema were widespread (supplemental Fig. 2, available at www. jneurosci.org as supplemental material).

Thus, there were three distinct temporal phases of injury in 
CONTROL
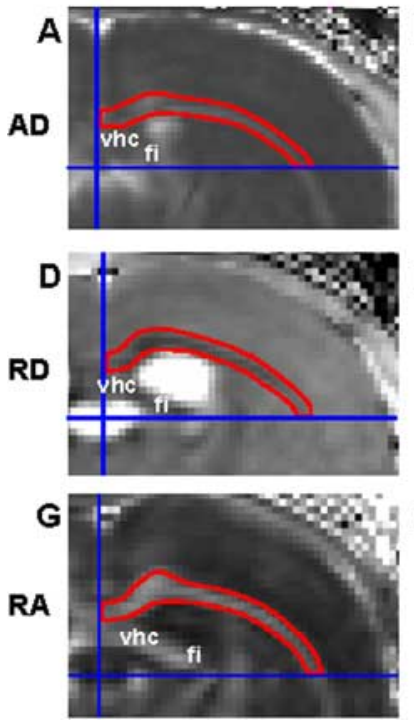

TBI: Acute
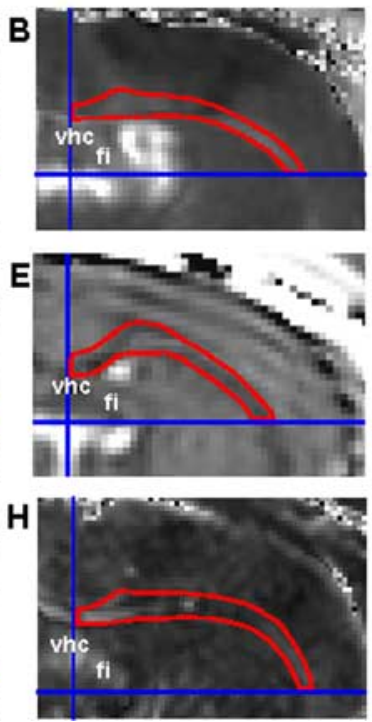

TBI: Subacute
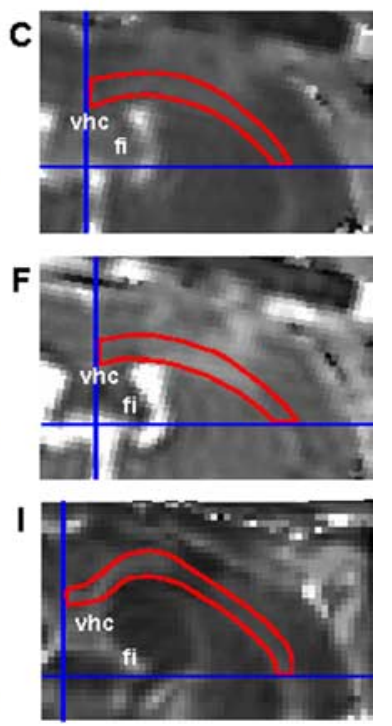

Figure 4. DTI signal characteristics at acute and subacute time points after TBI. The ROI is outlined in red, and the anatomical boundaries used to define the ROI are marked in blue. At this level, the medial boundary was the midline, bisecting the ventral hippocampal commissure (vhc), and the inferolateral boundary was defined by a horizontal line through the inferior edge of the fimbria (fi). Lighter grayscale shading indicates increased anisotropy or greater diffusivity. $A, A D$ was elevated in the uninjured corpus callosum and external capsule compared with surrounding cortical and subcortical gray matter. $\boldsymbol{B}, A$ cutely after TBI, AD was reduced within the white matter. Shown is a pericontusional region rostral to the epicenter of the injury [bregma, $-0.46 \mathrm{~mm}$ (Franklin and Paxinos, 1997)]. C, Subacutely, AD appeared to normalize, although parts of the overlying cortex had an elevated signal. $\boldsymbol{D}, \mathrm{RD}$ was reduced in the uninjured white matter relative to gray matter. Ventricles and periventricular regions appear bright on this image. $\boldsymbol{E}$, Acutely after TBI, RD was little changed in the white matter. $\boldsymbol{F}$, Subacutely, RD was markedly elevated in the white matter, and portions of the overlying cortex also showed high signal. G, RA was markedly elevated in uninjured white matter relative to gray matter. $\boldsymbol{H}$, After acute injury, RA in white matter was diminished dramatically. $\boldsymbol{I}$, Subacutely, RA was still strongly reduced. Gray matter regions showed low RA in control and at all time points after injury. Examples are shown for illustrative purposes and are not necessarily from the same mouse.

pericontusional white matter that can be characterized as follows: in the first $24 \mathrm{~h}$, the predominant pathology was relatively pure axonal injury without gliosis or demyelination. In the later acute stage (4 d), axonal injury was still marked, but gliosis became pronounced. In the subacute stage ( $7 \mathrm{~d}$ to 1 month), axonal injury became less apparent, myelin thinning along with active demyelination by macrophages was prevalent, and gliosis remained prominent.

\section{DTI of traumatic axonal injury}

Based on these histological and EM results, we hypothesized that DTI at $4-6 \mathrm{~h}, 24 \mathrm{~h}$, and $4 \mathrm{~d}$ would show a decrease in AD attributable to extensive axonal injury (axonal injury would be expected to cause a reduction in diffusion parallel to the predominant fiber direction of the axons). This would, in turn, cause a marked reduction in RA and a modest reduction in mean diffusivity or ADC. Our previous results at $24 \mathrm{~h}$ after TBI are consistent with this hypothesis (Mac Donald et al., 2007). At the subacute time points, we hypothesized that there would be an increase in RD attributable to demyelination, which would be expected to reduce barriers of diffusion perpendicular to the predominant fiber direction of the axons. We also predicted concomitant mild reductions in $\mathrm{AD}$, as a result of persistent axonal injury. These changes would be expected to cause a continued reduction in RA and a modest net increase in mean diffusivity.

We used the same anatomical landmarks used to describe the ROIs for histological analysis to localize the regions of axonal injury on the MR images (Fig. 4, outlined in red). In control images, normal white matter was observed to have high AD (Fig.
4A), low RD (Fig. 4D), and high RA (Fig. $4 G$ ), as expected for a highly organized tissue. Acutely after injury, AD decreased relative to control (Fig. $4 B$ ). Surprisingly, this was not the case at the subacute time points; $\mathrm{AD}$ instead appeared to be comparable to control (Fig. $4 C$ ). In contrast, RD appeared normal acutely (Fig. $4 E$ ) and was increased subacutely (Fig. $4 F$ ). A reduction in RA within the ROI was noted at all time points after injury (Fig. $4 H, I$ ).

For quantitative analyses, the average signal intensities through the complete rostrocaudal ROI spanning nine coronal slices were calculated and compared between groups (Fig. 5). There was a statistically significant reduction in $\mathrm{AD}$ at each of the acute time points after injury compared with control (Fig. 5A). Subacutely, AD was not significantly different from control. This may represent a pseudonormalization given the axonal pathology still apparent at these time points (Figs. 1, 2 ). As a group, the subacute time points differed significantly from the pooled acute time points.

$\mathrm{RD}$ remained unchanged acutely and then became significantly elevated subacutely (Fig. 5B). A highly significant reduction in RA was noted at all time points after injury (Fig. 5C). The mean diffusivity overall was variably reduced or unchanged acutely, whereas it was variably elevated or unchanged subacutely (Fig. 5D). There were highly significant differences between pooled acute injury groups and pooled subacute injury groups in terms of $\mathrm{RD}$ and mean diffusivity but not RA.

DTI accurately categorizes individual mice into uninjured, acute injury, and subacute injury groups

In a clinical setting, significant differences between groups are insufficient for the assessment of an individual patient. Each scan must be correctly evaluated in isolation, so there should ideally be no overlap between diagnostic categories of interest. Of the imaging parameters evaluated (supplemental Fig. 3, available at www.jneurosci.org as supplemental material); only RA permitted a complete separation between injured and uninjured mice. In a scatter plot of the data from each mouse, there was no overlap between the RA values of the control and TBI groups (Fig. 6A); $\mathrm{RA}$ in the ROI was reduced in $100 \%$ of injured animals at all time points.

In addition, the acute and subacute injured groups could be reliably separated from each other using mean diffusivity (Fig. $6 A$ ), $\mathrm{AD}$ (supplemental Fig. $4 A$, available at www.jneurosci.org as supplemental material), or RD (supplemental Fig. $4 B$, available at www.jneurosci.org as supplemental material). Mean diffusivity, $\mathrm{RD}$, and $\mathrm{AD}$ were all consistently lower in acutely injured mice than in mice with subacute injuries. Twenty-one of 23 (91\%) injured mice could be correctly categorized into acute or subacute injury, and 27 of 29 (93\%) mice overall could be correctly categorized into uninjured, acute injury, or subacute injury. Thus, DTI was not only able to distinguish injured from 
A
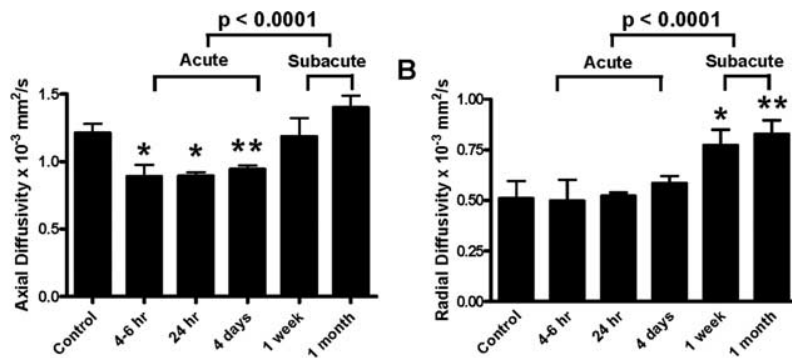

C

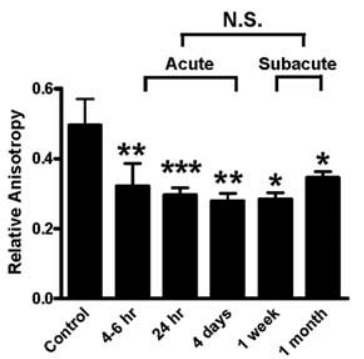

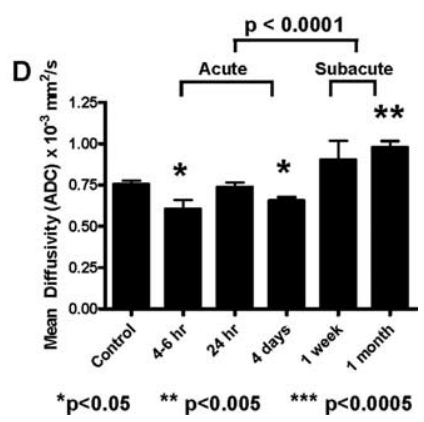

Figure 5. Quantitative analysis of DTI parameters after TBI. $\boldsymbol{A}$, Changes in average AD over time across the complete ROl. AD was significantly reduced at the acute time points ( $4-6 \mathrm{~h}$, $24 \mathrm{~h}, 4 \mathrm{~d}$ ) after injury and increased (pseudo-normalized) at the subacute time points (1 week, 1 month). Values at pooled acute time points were significantly different from those at pooled subacute times. $\boldsymbol{B}$, Changes in average RD over time. RD remained within the normal range until 1 week after TBI, when it became significantly elevated. Again, the values at subacute time points were significantly different from those at the acute time points. $\boldsymbol{C}$, Changes in RA over time. Highly significant reductions in RA were noted at all acute and subacute time points. $\boldsymbol{D}$, Changes in mean diffusivity over time. Although there were not consistent changes with respect to control, acute and subacute injuries differed markedly from each other. $n=4-6$ mice per group. Statistical significance was determined with a Student's $t$ test for independent samples. Error bars represent SDs.

uninjured white matter but also yielded reliable information about the approximate timing of injury.

We next asked whether the extent of DTI signal changes correlated with the severity of histologically visualized axonal injury, as assessed using quantitative stereology. There were statistically significant correlations of moderate strength between both APPand neurofilament-stained axonal varicosity counts and several DTI parameters (supplemental Figs. 5, 6, available at www. jneurosci.org as supplemental material). There were no significant correlations between the numbers of GFAP-immunoreactive astrocytes and any of the MR imaging parameters (supplemental Fig. 7, available at www.jneurosci.org as supplemental material). This, and the finding that GFAP-immunoreactive astrocytes increased dramatically from 1 to $4 \mathrm{~d}$ after injury without a clear corresponding MRI change, allowed us to infer that the signal abnormalities observed with DTI were not indicative of gliosis.

\section{Discussion}

In summary, we have demonstrated that DTI is capable of detecting pericontusional white matter injury in a mouse model of TBI at a range of acute to subacute time points. DTI appears more sensitive than conventional MRI for this purpose at all of the time points studied. Furthermore, DTI offers information on the timing of injury such that subacute injuries ( $7 \mathrm{~d}$ to 1 month old) can be differentiated from acute injuries ( $\leq 4 \mathrm{~d}$ old) with a high degree of accuracy at the level of the individual scan. This differentiation was possible because of the stereotypical evolution of the injury: predominantly axonal injury at acute time points and
A
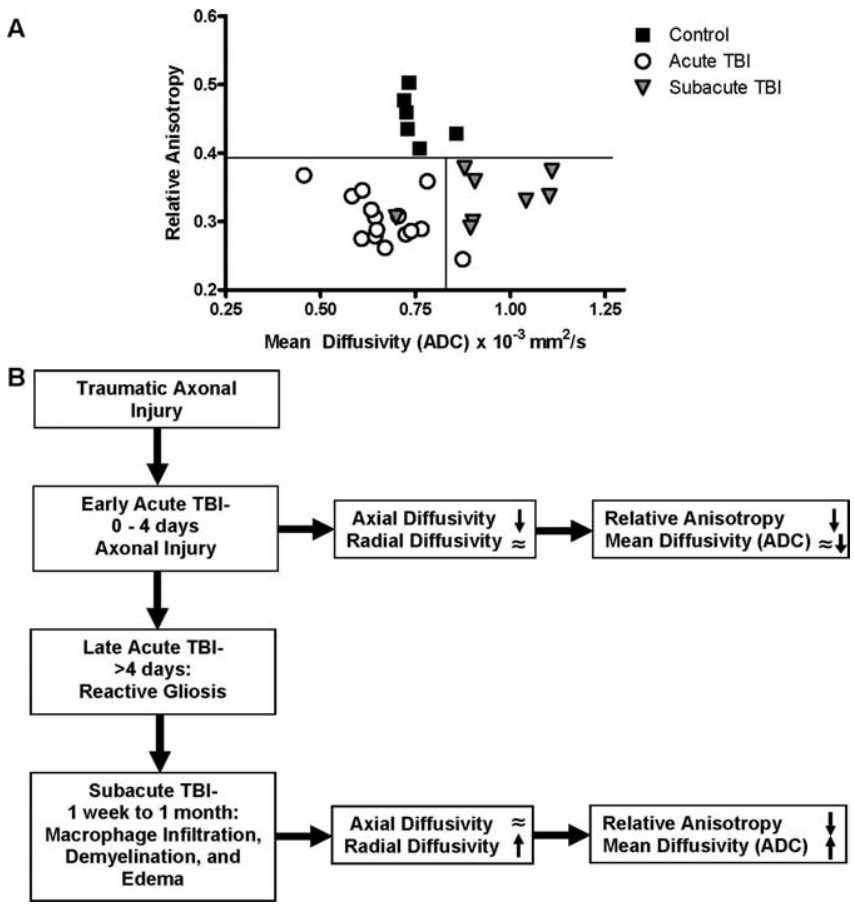

Figure 6. Discriminative value of DTI.A, Scatterplot of RA versus mean diffusivity for control, acute, and subacute time points. There was no overlap between the control and injured groups in terms of RA, and there was very little overlap between the acute and subacute injury groups in terms of mean diffusivity. $\boldsymbol{B}$, Schematic of changes after traumatic axonal injury over time and the corresponding DTI characteristics. During the early acute phase after TBI (4-6h, $24 \mathrm{~h}$ ), axonal injury is present histologically, and $A D$ is reduced. This in turn causes a reduction in $R A$ and a slight reduction in mean diffusivity. By $4 \mathrm{~d}$ after injury, reactive gliosis is present histologically, but there are no accompanying DTI changes. At 1 week to 1 month after injury, there is significant macrophage infiltration, demyelination, and edema. These changes lead to an elevation in both axial diffusivity and $\mathrm{RD}$, which in turn causes a reduction in RA and an increase in mean diffusivity.

primarily demyelination and edema at subacute time points (Fig. 6B).

Critical next steps will be to determine the extent to which the time course of signal abnormalities seen in this mouse model of relatively severe, pericontusional white matter injury applies to milder injuries, diffuse injures not adjacent to a contusion, and white matter injuries in human TBI patients. Encouragingly, the evolution of the injury over time as assessed with immunohistochemistry and electron microscopy in our mouse model is similar to that observed in autopsy series from human TBI patients. In humans, axonal injury with similar histological features has been reported days to months after trauma, with the highest prevalence around $24 \mathrm{~h}$ after injury (Adams et al., 1977, 1982, 1989; Vanezis et al., 1987; Christman et al., 1994; Graham et al., 1995; Geddes et al., 1997, 2000; Oehmichen et al., 1999). Reactive astrocytes can be seen as early as $1 \mathrm{~d}$ after TBI, but their numbers and GFAP immunoreactivity increase markedly during the first week (Vanezis et al., 1987; Geddes et al., 1997; Geddes et al., 2000; Hausmann et al., 2000). Infiltration of macrophages occurs at 3-5 d after trauma and is most marked 1-2 weeks after injury (Adams et al., 1977; Geddes et al., 1997; Oehmichen et al., 1999). Other strengths and limitations of this mouse model and the specific histological techniques used have previously been addressed (Mac Donald et al., 2007).

However, there are areas in which the human literature does not provide clear information on the evolution of these injuries. One example involves the timing of demyelination in human TBI 
(Strich, 1956, 1961; Nevin, 1967; Oehmichen et al., 2003). Another issue is whether pericontusional axonal injury (Strich, 1961; Cervos-Navarro and Lafuente, 1991; Hausmann and Betz, 2000; Hausmann et al., 2000) differs fundamentally from diffuse axonal injury not associated with contusion (Adams et al., 1977, 1982; Vanezis et al., 1987; Graham et al., 1995; Geddes et al., 1997). The controlled cortical impact model used in this study produces a central contusion and surrounding pericontusional axonal injury. This potentially differs from diffuse axonal injury that occurs in a scattered, multifocal distribution. Whether such differences are important remains an open question and have been discussed in detail previously (Mac Donald et al., 2007). Again encouragingly, DTI findings similar to ours have been reported in an animal model of diffuse, as opposed to pericontusional axonal injury at a single acute time point (Zakaria et al., 2007). It is likely that diffuse and pericontusional axonal injury will have similar features at acute time points before substantial Wallerian degeneration has occurred. At subacute time points, pericontusional Wallerian degeneration secondary to cell loss within the contusion itself may affect the DTI signals, and so the time course of DTI signal change in models of diffuse axonal injury without Wallerian degeneration is an important topic for future investigation.

It was interesting that $\mathrm{AD}$ in our model appeared to undergo a pseudo-normalization, returning to normal values despite the continued presence of axonal injury 1 week to 1 month after injury. An increase in overall diffusivity attributable to edema or other factors appears to be responsible for this trend. Conventional diffusion-weighted imaging (DWI) studies have shown the sensitivity of this MRI sequence for the detection of cerebral infarction varies as a function of time after injury because of a similar pseudo-normalization. At early stages, DWI is more sensitive than $\mathrm{T} 2$, but at subacute stages, $\mathrm{T} 2$ becomes more sensitive than DWI as the diffusion-weighted images undergo pseudonormalization (Schlaug et al., 1997; Copen et al., 2001; Schaefer et al., 2005). Similar changes in DWI signal characteristics over time after TBI have been observed in rats (Alsop et al., 1996; Albensi et al., 2000; Van Putten et al., 2005), pigs (Duhaime et al., 2003), and humans (Pasco et al., 2006). However, these studies did not use DTI and did not focus on white matter injury.

In contrast to $\mathrm{AD}, \mathrm{RA}$ was markedly abnormal at all of the time points studied and did not undergo pseudo-normalization. This measure appears to be very sensitive overall to the presence of white matter injury. Although RA varies considerably under control condition from region to region within white matter, abnormalities within specific regions can still be detected reliably by comparison with normal values for each region (Mac Donald et al., 2007). In contrast, mean diffusivity is more homogeneous across white matter regions but was found in this model to be less sensitive than RA, especially at acute time points, to pericontusional traumatic axonal injury.

For practical use as a diagnostic indicator, measures of anisotropy such as RA and fractional anisotropy would need to be compared with normal values for specific white matter regions. As this technique becomes widely used, clinical radiologists will likely become expert at distinguishing normal from abnormal anisotropy on a region-by-region basis, just as they are at distinguishing normal from abnormal T1 and T2 signals. If the injury is primarily unilateral, comparison with uninjured contralateral regions could be performed (Mac Donald et al., 2007), although caution should be used in applying this method to midline white matter structures. Alternatively, off-line postprocessing anatomical coregistration with age-matched normal controls could be performed, and a resultant statistical map of anisotropy abnormalities could be displayed (Wieshmann et al., 1999).

If the evolution of DTI signal changes over time occurs in human TBI patients in a stereotypical manner similar to that described here in our mouse model, the ability to assess the expected changes in the signal characteristics of the lesions over time could add further utility. As noted above, many further detailed studies in experimental and clinical settings will be required to establish this, but several motivating examples are worth noting. For therapeutic trials, the effect of an investigational agent on the severity of axonal injury could be determined by comparing the observed DTI signal characteristics after treatment with the expected evolution of DTI signal changes. Using DTI as a biomarker for axonal injury could markedly improve the efficiency of such trials. In a forensic investigation, the approximate age of a pericontusional traumatic white matter lesion could be assessed to either confirm or call into question the reports of witnesses or other evidence (Case et al., 2001; Oehmichen et al., 2003). A growing body of forensic imaging literature supports this possibility (Kahana and Hiss, 1999; Thali et al., 2007).

Thus, DTI has tremendous potential as a clinical tool in the assessment of TBI, as well as many other illnesses affecting white matter such as multiple sclerosis, ischemic stroke, HIV/AIDS dementia, Alzheimer's disease, amyotropic lateral sclerosis, traumatic spinal cord injury, and inherited leukoencephalopathies. However, many aspects of this technique still require validation. Our work is significant because it provides one such validation: a direct, quantitative comparison of DTI with pathologically verified pericontusional axonal injury at multiple time points after trauma.

\section{References}

Adams H, Mitchell DE, Graham DI, Doyle D (1977) Diffuse brain damage of immediate impact type. Its relationship to "primary brain-stem damage" in head injury. Brain 100:489-502.

Adams JH, Graham DI, Murray LS, Scott G (1982) Diffuse axonal injury due to nonmissile head injury in humans: an analysis of 45 cases. Ann Neurol 12:557-563.

Adams JH, Doyle D, Ford I, Gennarelli TA, Graham DI, McLellan DR (1989) Diffuse axonal injury in head injury: definition, diagnosis and grading. Histopathology 15:49-59.

Albensi BC, Knoblach SM, Chew BG, O’Reilly MP, Faden AI, Pekar JJ (2000) Diffusion and high resolution MRI of traumatic brain injury in rats: time course and correlation with histology. Exp Neurol 162:61-72.

Alsop DC, Murai H, Detre JA, McIntosh TK, Smith DH (1996) Detection of acute pathologic changes following experimental traumatic brain injury using diffusion-weighted magnetic resonance imaging. J Neurotrauma 13:515-521.

Arfanakis K, Haughton VM, Carew JD, Rogers BP, Dempsey RJ, Meyerand ME (2002) Diffusion tensor MR imaging in diffuse axonal injury. AJNR Am J Neuroradiol 23:794-802.

Assaf Y, Beit-Yannai E, Shohami E, Berman E, Cohen Y (1997) Diffusionand T2-weighted MRI of closed-head injury in rats: a time course study and correlation with histology. Magn Reson Imaging 15:77-85.

Brody DL, Mac Donald C, Kessens CC, Yuede C, Parsadanian M, Spinner M, Kim E, Schwetye KE, Holtzman DM, Bayly PV (2007) Electromagnetic controlled cortical impact device for precise, graded experimental traumatic brain injury. J Neurotrauma 24:657-673.

Case ME, Graham MA, Handy TC, Jentzen JM, Monteleone JA (2001) Position paper on fatal abusive head injuries in infants and young children. Am J Forensic Med Pathol 22:112-122.

Cervos-Navarro J, Lafuente JV (1991) Traumatic brain injuries: structural changes. J Neurol Sci [Suppl] 103:S3-S14.

Christman CW, Grady MS, Walker SA, Holloway KL, Povlishock JT (1994) Ultrastructural studies of diffuse axonal injury in humans. J Neurotrauma 11:173-186.

Copen WA, Schwamm LH, Gonzalez RG, Wu O, Harmath CB, Schaefer PW, Koroshetz WJ, Sorensen AG (2001) Ischemic stroke: effects of etiology 
and patient age on the time course of the core apparent diffusion coefficient. Radiology 221:27-34.

Duhaime AC, Hunter JV, Grate LL, Kim A, Golden J, Demidenko E, Harris C (2003) Magnetic resonance imaging studies of age-dependent responses to scaled focal brain injury in the piglet. J Neurosurg 99:542-548.

Franklin KB, Paxinos G (1997) The mouse brain in stereotaxic coordinates. San Diego: Academic.

Geddes JF, Vowles GH, Beer TW, Ellison DW (1997) The diagnosis of diffuse axonal injury: implications for forensic practice. Neuropathol Appl Neurobiol 23:339-347.

Geddes JF, Whitwell HL, Graham DI (2000) Traumatic axonal injury: practical issues for diagnosis in medicolegal cases. Neuropathol Appl Neurobiol 26:105-116.

Gennarelli TA, Thibault LE, Adams JH, Graham DI, Thompson CJ, Marcincin RP (1982) Diffuse axonal injury and traumatic coma in the primate. Ann Neurol 12:564-574.

Graham DI, Adams JH, Nicoll JA, Maxwell WL, Gennarelli TA (1995) The nature, distribution and causes of traumatic brain injury. Brain Pathol 5:397-406.

Hanstock CC, Faden AI, Bendall MR, Vink R (1994) Diffusion-weighted imaging differentiates ischemic tissue from traumatized tissue. Stroke 25:843-848.

Hausmann R, Betz P (2000) The time course of the vascular response to human brain injury-an immunohistochemical study. Int J Legal Med 113:288-292.

Hausmann R, Riess R, Fieguth A, Betz P (2000) Immunohistochemical investigations on the course of astroglial GFAP expression following human brain injury. Int J Legal Med 113:70-75.

Huisman TA, Schwamm LH, Schaefer PW, Koroshetz WJ, Shetty-Alva N, Ozsunar Y, Wu O, Sorensen AG (2004) Diffusion tensor imaging as potential biomarker of white matter injury in diffuse axonal injury. AJNR Am J Neuroradiol 25:370-376.

Inglese M, Makani S, Johnson G, Cohen BA, Silver JA, Gonen O, Grossman RI (2005) Diffuse axonal injury in mild traumatic brain injury: a diffusion tensor imaging study. J Neurosurg 103:298-303.

Kahana T, Hiss J (1999) Forensic radiology. Br J Radiol 72:129-133.

King Jr JT, Carlier PM, Marion DW (2005) Early Glasgow Outcome Scale scores predict long-term functional outcome in patients with severe traumatic brain injury. J Neurotrauma 22:947-954.

Kochanek PM, Marion DW, Zhang W, Schiding JK, White M, Palmer AM, Clark RS, O’Malley ME, Styren SD, Ho C, DeKosky ST (1995) Severe controlled cortical impact in rats: assessment of cerebral edema, blood flow, and contusion volume. J Neurotrauma 12:1015-1025.

MacDonald CL, Dikranian K, Song SK, Bayly PV, Holtzman DM, Brody DL (2007) Detection of traumatic axonal injury with diffusion tensor imaging in a mouse model of traumatic brain injury. Exp Neurol 205:116-131.

Naganawa S, Sato C, Ishihra S, Kumada H, Ishigaki T, Miura S, Watanabe M, Maruyama K, Takizawa O (2004) Serial evaluation of diffusion tensor brain fiber tracking in a patient with severe diffuse axonal injury. AJNR Am J Neuroradiol 25:1553-1556.

Nakayama N, Okumura A, Shinoda J, Yasokawa YT, Miwa K, Yoshimura SI, Iwama T (2006) Evidence for white matter disruption in traumatic brain injury without macroscopic lesions. J Neurol Neurosurg Psychiatry $77: 850-855$.

Nevin NC (1967) Neuropathological changes in the white matter following head injury. J Neuropathol Exp Neurol 26:77-84.

Oehmichen M, Theuerkauf I, Meissner C (1999) Is traumatic axonal injury (AI) associated with an early microglial activation? Application of a double-labeling technique for simultaneous detection of microglia and AI. Acta Neuropathol (Berl) 97:491-494.

Oehmichen M, Walter T, Meissner C, Friedrich HJ (2003) Time course of cortical hemorrhages after closed traumatic brain injury: statistical analysis of posttraumatic histomorphological alterations. J Neurotrauma 20:87-103.

Oppenheimer DR (1968) Microscopic lesions in the brain following head injury. J Neurol Neurosurg Psychiatry 31:299-306.

Pasco A, Ter Minassian A, Chapon C, Lemaire L, Franconi F, Darabi D, Caron C, Benoit JP, Le Jeune JJ (2006) Dynamics of cerebral edema and the apparent diffusion coefficient of water changes in patients with severe traumatic brain injury. A prospective MRI study. Eur Radiol 16:1501-1508.

Pilz P (1983) Axonal injury in head injury. Acta Neurochir Suppl (Wien) 32:119-123.

Povlishock JT, Erb DE, Astruc J (1992) Axonal response to traumatic brain injury: reactive axonal change, deafferentation, and neuroplasticity. J Neurotrauma 9 [Suppl 1]:S189-S200.

Ptak T, Sheridan RL, Rhea JT, Gervasini AA, Yun JH, Curran MA, Borszuk P, Petrovick L, Novelline RA (2003) Cerebral fractional anisotropy score in trauma patients: a new indicator of white matter injury after trauma. AJR Am J Roentgenol 181:1401-1407.

Rugg-Gunn FJ, Symms MR, Barker GJ, Greenwood R, Duncan JS (2001) Diffusion imaging shows abnormalities after blunt head trauma when conventional magnetic resonance imaging is normal. J Neurol Neurosurg Psychiatry 70:530-533.

Schaefer PW, Copen WA, Lev MH, Gonzalez RG (2005) Diffusionweighted imaging in acute stroke. Neuroimaging Clin N Am 15:503-530, ix-x.

Schlaug G, Siewert B, Benfield A, Edelman RR, Warach S (1997) Time course of the apparent diffusion coefficient (ADC) abnormality in human stroke. Neurology 49:113-119.

Silver JA, McAllister TW, Yudofsky SC (2005) Textbook of traumatic brain injury, Ed 1. Arlington, VA: American Psychiatric Publishing.

Smith DH, Meaney DF, Shull WH (2003) Diffuse axonal injury in head trauma. J Head Trauma Rehabil 18:307-316.

Song SK, Sun SW, Ju WK, Lin SJ, Cross AH, Neufeld AH (2003) Diffusion tensor imaging detects and differentiates axon and myelin degeneration in mouse optic nerve after retinal ischemia. NeuroImage 20:1714-1722.

Song SK, Kim JH, Lin SJ, Brendza RP, Holtzman DM (2004) Diffusion tensor imaging detects age-dependent white matter changes in a transgenic mouse model with amyloid deposition. Neurobiol Dis 15:640-647.

Sterio DC (1984) The unbiased estimation of number and sizes of arbitrary particles using the disector. J Microsc 134:127-136.

Strich SJ (1956) Diffuse degeneration of the cerebral white matter in severe dementia following head injury. J Neurol Neurosurg Psychiatry 19:163-185.

Strich SJ (1961) Shearing of nerve fibers as a cause of brain damage due to head injury. Lancet 2:443-448.

Thali MJ, Jackowski C, Oesterhelweg L, Ross SG, Dirnhofer R (2007) VIRTOPSY - the Swiss virtual autopsy approach. Leg Med (Tokyo) 9:100-104.

Vanezis P, Chan KK, Scholtz CL (1987) White matter damage following acute head injury. Forensic Sci Int 35:1-10.

Van Putten HP, Bouwhuis MG, Muizelaar JP, Lyeth BG, Berman RF (2005) Diffusion-weighted imaging of edema following traumatic brain injury in rats: effects of secondary hypoxia. J Neurotrauma 22:857-872.

Voss HU, Uluc AM, Dyke JP, Watts R, Kobylarz EJ, McCandliss BD, Heier LA, Beattie BJ, Hamacher KA, Vallabhajosula S, Goldsmith SJ, Ballon D, Giacino JT, Schiff ND (2006) Possible axonal regrowth in late recovery from the minimally conscious state. J Clin Invest 116:2005-2011.

Werring DJ, Clark CA, Barker GJ, Miller DH, Parker GJ, Brammer MJ, Bullmore ET, Giampietro VP, Thompson AJ (1998) The structural and functional mechanisms of motor recovery: complementary use of diffusion tensor and functional magnetic resonance imaging in a traumatic injury of the internal capsule. J Neurol Neurosurg Psychiatry 65:863-869.

Wieshmann UC, Symms MR, Clark CA, Lemieux L, Parker GJ, Barker GJ, Shorvon SD (1999) Blunt-head trauma associated with widespread water-diffusion changes. Lancet 353:1242-1243.

Wilde EA, Chu Z, Bigler ED, Hunter JV, Fearing MA, Hanten G, Newsome MR, Scheibel RS, Li X, Levin HS (2006) Diffusion tensor imaging in the corpus callosum in children after moderate to severe traumatic brain injury. J Neurotrauma 23:1412-1426.

Zakaria N, Kallakuri S, Cavanaugh J, Shen Y, Yu Y, Kou Z, Haacke M (2007) Assessing the potential of diffusion tensor imaging as a non-invasive tool for detecting diffuse axonal injuries in a rodent model of traumatic brain injury. J Neurotrauma 24:1233. 